\title{
Tool for Guidance: Evidence-Based Recommendations for Managing Febrile Neutropenia
}

\author{
Andrew J. Ullmann \\ Department of Hematology, Oncology and Pneumology, Johannes Gutenberg-University Medical Center, Mainz, Germany
}

\section{See Article on Page 220-252}

Physicians face a number of challenges when developing guidelines, as the context in which we practice medicine is ever changing. In this edition of the Korean Journal of Internal Medicine, Korean experts in the fields of infectious disease, hematology-oncology, laboratory medicine, internal medicine, and methodology took that challenge. Their task was to develop evidencebased guidelines for febrile neutropenia [1]. Febrile neutropenia is a consequence of collateral damage caused by chemotherapy for an underlying hematological disease and has a high mortality rate that clinicians must tackle.

These guidelines are very helpful, as they provide practical guidance for clinicians that will help young physicians as well as more experienced physicians. The guidelines consist of text and tables for decision making and incorporate dosing recommendations and practical figures with algorithms to follow. This is not always seen in other guidelines [2]. Both the process of developing these Korean guidelines and the means by which the results were obtained were made very transparent which is not always provided by others. Another positive aspect of these guidelines is that the grading system used is identical to that used by the Infectious Diseases Society of America (ISDA) [2,3]. This allows the foreign reader to understand the strength of the recommendations and the quality of the evidence. These Korean guidelines will be discussed widely in the academic world because they provide additional insights into the clinical care of patients with febrile neutropenia.
In the development of given guidelines, it is important to remember who is addressed. American or European guidelines might not adequately represent or even address the same issues as those that occur in Korea. The epidemiology might differ, and the approval and availability of certain drugs has to be considered. This cannot be provided by outside guidelines. Regional guidelines (e.g., European, Asian-Pacific, or Australian) might provide physicians with some insight, but they will always need to be adjusted for the respective countries. However, I would like to offer one word of caution, which is to point out the necessity of ensuring that guidelines are updated on a regular basis.

What can I say about the quality of the guidelines? Today, certain qualities are required: the scope and purpose of the guidelines, followed by stakeholder involvement, rigor of development, clarity of presentation, applicability, and editorial independence [4]. These guidelines have met these criteria. Interestingly, besides the authors themselves, advice from other Korean experts in this field of expertise was incorporated via a questionnaire (full data not presented in the publication but available from the corresponding author) [1] .

Funding from industry played no role in these guidelines and was not involved during their development. This is important for guaranteeing independence, which is not provided when representatives of companies are present. Even their simply sitting in as learning observers is considered inappropriate. Obviously, this did not occur with these guidelines.

When considering patient care, it is also important to understand the limitations that national approval places on the use of treatments, such as anti-infective agents. 
This always needs to be considered in national guidelines. During the development of guidelines, it is therefore essential to ask three simple questions repeatedly: What do clinicians want? Which option is better for patients? Is the chosen option truly better? [5] Understandably, physicians cannot change national legislation, but government officials should consider their input. On another note, the potential cost implications of applying the recommendations were considered in these guidelines.

It would be inappropriate for me discuss certain recommendations that I might or might not agree with. Nevertheless, it is pivotal to mention that the scientific process involved in the development of these guidelines was sound. The authors offer evidence-based recommendations by conducting an evaluation of available data and reviewed their importance for Korea. This evaluation was not influenced by any industry involvement and was reviewed by the authors' peers, who were allowed to comment via a questionnaire. This process is fairly unique in the world of guidelines, and others should follow in the footsteps of these Korean experts.

In summary, I would like to applaud the organizer, the National Evidence-based Healthcare Collaborating Agency, together with a team of experts from various Korean academic societies for completing this endeavor in an amazingly short time. These guidelines are very clinically orientated and practical, and they will hold medical care in this specific field of medicine to a high standard. (Korean J Intern Med 2011;26:135-136)

\section{Conflict of interest}

No potential conflict of interest relevant to this article was reported.

\section{REFERENCES}

1. Lee DG, Kim SH, Kim SY, et al. Evidence-based guidelines for empirical therapy of neutropenic fever in Korea. Korean J Intern Med 2011;26:220-252.

2. Freifeld AG, Bow EJ, Sepkowitz KA, et al. Clinical practice guideline for the use of antimicrobial agents in neutropenic patients with cancer: 2010 Update by the Infectious Diseases Society of America. Clin Infect Dis 2011;52:427-431.

3. The periodic health examination. Canadian Task Force on the Periodic Health Examination. Can Med Assoc J 1979;121:11931254 .

4. The AGREE Next Steps Consortium. The AGREE II Instrument [Internet]. The AGREE Research Trust, 2009 [cited 2011 May 15]. Available from: http://www.agreetrust.org.

5. Brozek JL, Akl EA, Compalati E, et al. Grading quality of evidence and strength of recommendations in clinical practice guidelines Part 3 of 3: the GRADE approach to developing recommendations. Allergy 2011;66:588-595. 